Pacific Journal of Mathematics

A SCHEME FOR APPROXIMATING BOUNDED ANALYTIC 


\section{A SCHEME FOR APPROXIMATING BOUNDED ANALYTIC FUNCTIONS ON CERTAIN SUBSETS OF THE UNIT DISC}

\section{A. STRAY}

We denote by $H^{\infty}$ the space of all bounded analytic functions in the unit disc $D=\{z:|z|>1\}$. We consider a relatively closed subset $S$ of $D$ with the following property: If $f \in H^{\infty}$ and its restriction $\left.f\right|_{S}$ to $S$ is uniformly continuous, there exists a bounded sequence of polynomials $\left\{p_{n}\right\}$ such that

(i) $p_{n} \rightarrow f$ uniformly on compact subsets of $D$.

(ii) $p_{n} \rightarrow f$ uniformly on $S$.

A set $S$ with this property, is called a Mergelyan set for $H^{\infty}$. The object of this paper is to give a new and constructive proof of the following result:

Theorem. Let $S$ be a relatively closed subset of $D$. Assume that the clusterpoints of $S$ on the unit circle $T=$ $\{z:|z|=1\}$ which are not in the nontangential closure of $S$, has zero linear measure. Then $S$ is a Mergelyan set for $H^{\infty}$.

A point $\eta \in T$ is said to be in the nontangential closure of $S$ if $\eta$ is a limit point of $S \cap D(\lambda, \eta)$ for some $\lambda>0$ where $D(\lambda, \eta)=$ $\{z \in D:|z-\eta|<\lambda(1-|z|)\}$.

The theorem above solves a problem raised by J. Detraz [1]. (She also proved the converse of the theorem.)

The first proof of it (due to A. M. Davie) was based on functional analysis, and is rather short and elegant. See [5] for details. But from a constructive point of view, the proof is not very satisfactory. So the main reason for introducing this new and longer proof, is that it gives a rather explicit construction of the approximating polynomials $\left\{p_{n}\right\}_{n=1}^{\infty}$.

This theorem is part of a more general problem in simultaneous approximation introduced to me by Professor L. Rubel. (See [4], [5], and [6].)

Proof of the theorem. We denote by $S^{2}$ the extended complex plane. If $B \subset S^{2}$, and $g$ is a function defined on $B$, then we define $\|g\|_{B}=\sup \{|g(z)|, z \in B\}$. Various absolute constants will be denoted by $A_{1}, A_{2}, \cdots$.

Let $f$ and $S$ be as in the above theorem. We assume $\|f\|_{D} \leqq 1$. 
If $E \subset T$ is measurable, $|E|$ denotes its linear measure. Let $D(\lambda, \rho)$ be as above. By a well known result of Fatou, ([3], page 34) there is a set $F \subset T$, with $|F|=0$, such that

$$
\lim _{\substack{z \rightarrow \rho \\ z \in D(\lambda, \rho)}} f(z) \stackrel{\text { def }}{=} f^{*}(\rho)
$$

exists for all $\rho \in T \backslash F$ and all $\lambda>0$. Let $E$ denote the limitpoints of $S$ on $T$. By (1) and our hypothesis on $S$, the continuous extension of $f$ to $S \cup E$, coincides with $f^{*}$ almost everywhere on $E$. (With respect to linear measure.)

By approximating as in $\S 1$ in [7], we can also assume that $f^{*}$ is continuous on $T \backslash E$. But then $f^{*}$ (considered as a function on $T$ ) is continuous on $T \backslash(E \backslash B)$, where $B$ denotes the interior of $E$ relative to $T$.

The approximation of $f$ is now done in two steps. If $|E \backslash B|=0$, the first step can be omitted. So we assume $|E \backslash B|>0$.

The idea is to modify Vitushkin's scheme for rational approximation (see [2], page 210) to be suitable for our problem. We shall construct functions $f_{n}, n=1,2, \cdots$ such that $g=f-\sum_{1}^{\infty} f_{n}$ has the following two properties if $\varepsilon>0$ is given in advance:

$$
\|f-g\|_{S}<\varepsilon \text {. }
$$

(3) $g$ is holomorphic in $D$ and continuous in $D \cup T \backslash E_{0}$, where $E_{0} \subset E \backslash B$ is compact and $\left|E_{0}\right|=0$. Also $\|g\|_{D} \leqq A_{1}$.

Before we construct $\left\{f_{n}\right\}_{n=1}^{\infty}$, let us indicate the second and last step in the proof. Denote by $A(D)$, all uniformly continuous analytic functions in $D$. We choose functions $a$ and $b$ from $A(D)$ such that there is a neighborhood $V$ of $E_{0}$ in $C$ with the following property

$$
\left\{\begin{array}{l}
|a-f|<\varepsilon \cdot 2^{-1} \text { on } S \cap V . \\
\max \left\{\|a\|_{D^{\prime}}\|b\|_{D}\right\} \leqq 2 . \\
b=0 \text { on } E_{0} \text { and if } z \in S, \text { then } \\
|a(z)-f(z)|>2 \varepsilon \Rightarrow|1-b(z)|<\varepsilon .
\end{array}\right.
$$

The construction of $a$ and $b$ can be done as in [3], page 80. We partion $E_{0}$ into finitely many subsets $E_{1}, \cdots, E_{n}$ with pairwise disjoint neighborhoods $W_{1}, \cdots, W_{n}$, such that there are complex numbers $\lambda_{j} 1 \leqq j \leqq n$ with $\left|f-\lambda_{j}\right|<\varepsilon 2^{-2}$ on each $E_{j}$. For each $j$ we argue as on page 80 in [3] and find $b_{j} \in A(D)$ with $b_{j}=0$ on $E_{j}$ and $\operatorname{Re} b_{j}<0$ on $D \backslash E_{j}$. Then we define 


$$
a=\sum_{1}^{n} \lambda_{j} e^{N b_{j}}
$$

where $N$ is a sufficiently large number. Then $|a-f|<\varepsilon 2^{-1}$ in some neighborhood $V$ of $E_{0}$, and we choose $b \in A(D)$ with $b=0$ on $E_{0}$, $\|b\|_{D} \leqq 1$ and $|1-b|<\varepsilon$ on $D \backslash V$.

Consider now $h=b(g-a)+a$. Then $h \in A(D)$ and since $g-h=$ $(g-a)(1-b)$, we have from (2), (3), and (4) above that $\|g-h\|_{S}<$ $\max \left\{\left(A_{1}+2\right) \varepsilon, 5 \varepsilon\right\}$. But then $\|f-h\|_{s}<A_{2} \varepsilon$, and we also have $\|h\|_{D} \leqq A_{3}$. Since $h \in A(D)$, this function is easy to approximate uniformly on $D$ by polynomials, and this completes the second step.

We turn to the construction of $\left\{f_{n}\right\}$.

Let $\varepsilon>0$ be given. We choose pairwise disjoint dises $A_{n}=$ $\Delta\left(w_{n}, \delta_{n}\right), n=1,2, \cdots$ and constants $t, M$, and $\lambda$ with the following properties

(5) $\quad w_{n} \in E \backslash B$ and there are numbers $\eta_{n}$ with $\delta_{n}<\eta_{n}<2 \delta_{n}<t$ such that $\left[\left(\Delta\left(w_{n}, \eta_{n}\right) \backslash \Delta\left(w_{n}, \delta_{n}\right)\right) \cap T\right] \subset T \backslash(E \backslash B)$.

(6) $\quad\left|E \backslash\left(B \cup\left(\bigcup_{n} \Delta_{n}\right)\right)\right|=0$.

(7) $\sup \left\{|f(z)-f(\rho)|, z, \rho \in \Delta\left(w_{n}, 2 \delta_{n}\right) \cap D\left(\lambda, w_{n}\right)\right\}<\varepsilon$.

(8) $\sup \{|f(z)-f(\rho)|, z, \rho \in S,|z-\rho| \leqq M t\}<\varepsilon$.

More details about how $t, \lambda$, and $M$ depend on $\varepsilon$, will be given below. The important thing is that they are given in advance, before $\left\{\Delta_{k}\right\}$ is constructed. The existence of $\left\{A_{k}\right\}$ satisfying (5)-(8) follows from Fatou's result (1) about nontangential limits mentioned above, and the fact that $E \backslash B$ is totally disconnected. To obtain (8) we use that $\left.f\right|_{S}$ is uniformly continuous.

We now choose numbers $r_{n}$ with $\delta_{n}<r_{n}<\eta_{n}$, and smooth functions $\varphi_{n}$ with support in $A\left(w_{n}, r_{n}\right)$ such that we also have for all $n$

$$
\begin{aligned}
& 0 \leqq \varphi_{n} \leqq 1, \quad \varphi_{n} \equiv 1 \text { near } \Delta_{n}, \quad \text { and } \\
& \left\|\frac{\partial \varphi_{n}}{\partial \bar{z}}\right\|_{C} \leqq A_{2}\left(\eta_{n}-\delta_{n}\right)^{-1} .
\end{aligned}
$$

We extend $f$ to $C$ by the equation $f(z)=f(1 / \bar{z})$. Define functions $G_{n}, n=1,2, \cdots$ by

$$
\begin{aligned}
G_{n}(\rho) & =T_{\varphi_{n}} f(\rho)=\frac{1}{\pi} \iint \frac{f(z)-f(\rho)}{z-\rho} \frac{\partial \varphi_{n}}{\partial \bar{z}} d x d y \\
& =\varphi_{n}(\rho) \cdot f(\rho)+\frac{1}{\pi} \iint \frac{f(z)}{z-\rho} \frac{\partial \varphi_{n}}{\partial \bar{z}} d x d y .
\end{aligned}
$$

For basic properties of the $T_{\varphi}$-operator we refer to [2], page 
30. These properties gives that $G_{n}$ is analytic in $D$ and continuous wherever $f$ is. Also $f-G_{n}$ is analytic near $\Delta_{n} \cap(E \backslash B)$. Since $\|f\|_{c} \leqq 1$, (9) shows that

$$
\left\|G_{n}\right\|_{C} \leqq A_{3}
$$

We also claim that the constants $t, M$, and $\lambda$ can be chosen so that

$$
\left\|G_{n}\right\|_{S}<A_{4} \varepsilon .
$$

To see this, note first that since $G_{n}$ is analytic in $S^{2} \backslash \Delta\left(w_{n}, t\right)$ and $G_{n}(\infty)=0$, an easy application of Schwartz lemma shows that $\left|G_{n}(\rho)\right|<A(M-1)^{-1}<\varepsilon$ if $M$ is sufficiently large, and $\left|\rho-w_{n}\right|>M t$. This does not contradict (8) if $t$ is sufficiently small. If we use (8), we see that if $p \in S$ and $\left|\rho-w_{n}\right| \leqq M t$, then we can estimate the first integral in $\left(^{*}\right)$ so that

$$
\left|G_{n}(\rho)\right| \leqq A_{5} \varepsilon+K(\lambda)
$$

where $K(\lambda)$ is a constant tending to zero if $\lambda \rightarrow \infty$. It is easy to see that we can choose $\lambda$ depending on $\varepsilon$, but not on $n$, such that $K(\lambda)<\varepsilon$. (The constant $K(\lambda)$ comes from integration over those $z \in \Delta\left(w_{n}, r_{n}\right)$ where $\left.|f(z)-f(\rho)|>2 \varepsilon\right)$.

We can now write $f_{n}$ as

$$
f_{n}=G_{n}-H_{n}
$$

where $H_{n}$ is a rational function with poles only in $\Delta\left(w_{n}, \delta_{n}\right) \backslash \bar{D}$ such that

$$
\begin{aligned}
& \left\|H_{n}\right\|_{S} \leqq\left\|G_{n}\right\|_{S} \leqq \varepsilon A_{6} \\
& \left\|H_{n}\right\|_{D} \leqq\left\|G_{n}\right\|_{D} \leqq A_{7} \\
& \left\|H_{n}-G_{n}\right\|_{S^{2} \backslash \Delta\left(w_{n}, \eta_{n}\right)}<\varepsilon 2^{-n} .
\end{aligned}
$$

The existence of $\left\{H_{n}\right\}$ is not quite trivial. If we map $\left(S^{2} \backslash \Delta\left(w_{n}, r_{n}\right)\right) \cup D$ conformally onto $D, S \cup\left(S^{2} \backslash \Delta\left(w_{n}, \eta_{n}\right)\right)$ is mapped to a Farrel set (see [5] for definition) in $D$, and the existence of $H_{n}$, $n=1,2, \cdots$, now follows from the main result in [5]. This completes the proof. In fact, the set $E_{0}$ in (3) above, is simply $\left(T \backslash\left(B \cup\left(\mathbf{U}_{n} \Delta_{n}\right)\right)\right.$.

Concluding remark. It is easy to verify that this proof applies to more general planar domains.

\section{REFERENCES}

1. J. Detraz, Algebres de fonctions analytique dans le disque, Ann. Sci. Ecole Norm. Sup., 3 (1970), 313-352. 
2. T. W. Gamelin, Uniform Algebras, Prentice Hall, Englewood Cliffs, N.J., 1969.

3. K. Hoffman, Banach Spaces of Analytic Functions, Prentice Hall, Englewood Cliffs, N.J., 1962.

4. L. A. Rubel, Symposium on Complex Analysis, Canterbury, 1973. Edited by J. Clunie and W. K. Hayman. Cambridge University Press.

5. A. Stray, Pointwise bounded approximation by functions satisfying a side conditions, Pacific J. Math., 51 (1974).

6. - Characterization of Mergelyan sets, Proc. Amer. Math. Soc., 44, number 2, (1974).

7. - Approximation and interpolation, Pacific J. Math., 40, number 2, (1972).

Received January 18, 1977 and in revised form August 24, 1977.
A. D. H.
P. O. Box 607
$4601 \mathrm{KR}$. SAND
NoRWAY 



\section{PACIFIC JOURNAL OF MATHEMATICS}

\section{EDITORS}

RICHARD ARENS (Managing Editor)

University of California

Los Angeles, California 90024

C. W. Curtis

University of Oregon

Eugene, OR 97403

C. C. MOORE

University of California

Berkeley, CA 94720

\section{J. DUGUNDJI}

Department of Mathematics University of Southern California Los Angeles, California 90007

R. Finn AND J. Milgram Stanford University Stanford, California 94305

ASSOCIATE EDITORS
E. F. BECKENBACH

B. H. NeUMANN

F. WOLF

K. YoshidA

\section{SUPPORTING INSTITUTIONS}

UNIVERSITY OF BRITISH COLUMBIA UNIVERSITY OF SOUTHERN CALIFORNIA CALIFORNIA INSTITUTE OF TECHNOLOGY STANFORD UNIVERSITY UNIVERSITY OF CALIFORNIA MONTANA STATE UNIVERSITY UNIVERSITY OF TOKYO UNIVERSITY OF NEVADA, RENO UNIVERSITY OF UTAH NEW MEXICO STATE UNIVERSITY OREGON STATE UNIVERSITY UNIVERSITY OF OREGON

WASHINGTON STATE UNIVERSITY UNIVERSITY OF WASHINGTON OSAKA UNIVERSITY 


\section{Pacific Journal of Mathematics}

\section{Vol. 74, No. $1 \quad$ May, 1978}

Gerald Arthur Anderson, Computation of the surgery obstruction groups

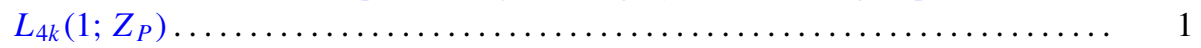

R. K. Beatson, The degree of monotone approximation ................ 5

Sterling K. Berberian, The character space of the algebra of regulated functions . . . 15

Douglas Michael Campbell and Jack Wayne Lamoreaux, Continua in the plane with

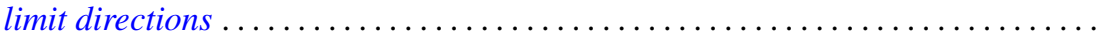

R. J. Duffin, Algorithms for localizing roots of a polynomial and the Pisot

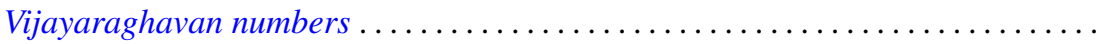

Alessandro Figà-Talamanca and Massimo A. Picardello, Functions that operate on

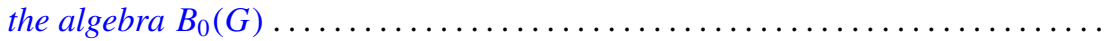

John Erik Fornaess, Biholomorphic mappings between weakly pseudoconvex

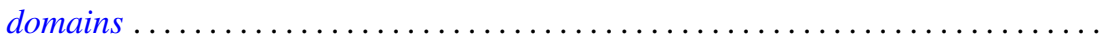

Andrzej Granas, Ronald Bernard Guenther and John Walter Lee, On a theorem of S.

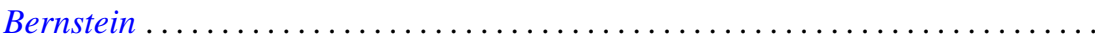

Jerry Grossman, On groups with specified lower central series quotients . .........

William H. Julian, Ray Mines, III and Fred Richman, Algebraic numbers, a constructive development . . . . . . . . . . . . . . . . . . . . . . .

Surjit Singh Khurana, A note on Radon-Nikodým theorem for finitely additive

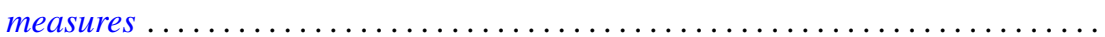

Garo K. Kiremidjian, A Nash-Moser-type implicit function theorem and nonlinear

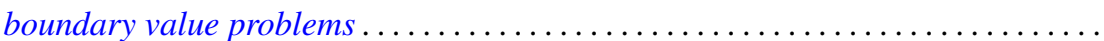

Ronald Jacob Leach, Coefficient estimates for certain multivalent functions ....

John Alan MacBain, Local and global bifurcation from normal eigenvalues. II . . 133

James A. MacDougall and Lowell G. Sweet, Three dimensional homogeneous algebras...

John Rowlay Martin, Fixed point sets of Peano continua ......

R. Daniel Mauldin, The boundedness of the Cantor-Bendixson order of some analytic sets...

Richard C. Metzler, Uniqueness of extensions of positive linear functions ..

Rodney V. Nillsen, Moment sequences obtained from restricted powers . .

Keiji Nishioka, Transcendental constants over the coefficient fields in differential elliptic function fields...

Gabriel Michael Miller Obi, An algebraic closed graph theorem

Richard Cranston Randell, Quotients of complete intersections by $\mathbf{C}^{*}$ actions . . 221

Bruce Reznick, Banach spaces which satisfy linear identities . .

Bennett Setzer, Elliptic curves over complex quadratic fields...

Arne Stray, A scheme for approximating bounded analytic functions on certain subsets of the unit disc.

Nicholas Th. Varopoulos, A remark on functions of bounded mean oscillation and bounded harmonic functions. Addendum to: "BMO functions and the

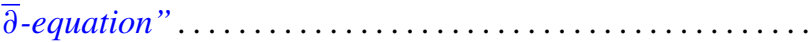

Charles Irvin Vinsonhaler, Torsion free abelian groups quasi-projective over their

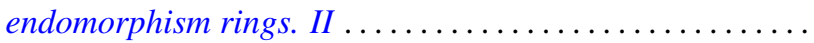

Thomas R. Wolf, Characters of $p^{\prime}$-degree in solvable groups ... 